\title{
DEVELOPMENT OF AN AUTOMATIC ICE FABRIC ANALYSER
}

\author{
by
}

\author{
Y. MORI, T. HONDOH
}

Department of Applied Physics, Faculty of Engineering, Hokkaido University, Sapporo, Japan 060

AND A. Higash

Division of Natural Sciences, International Christian University, Tokyo, Japan 181

\section{ABSTRACT}

An automatic ice fabric analyser has been developed which precisely determines both c-and a-axes of the individual grains in a thin polycrystalline ice specimen by the use of X-ray Laue method (eg Cullity 1956). The method of analysis is computer-aided indexing of the zone-axes in $\mathrm{X}$-ray Laue patterns. Crystal orientations can be automatically determined by the analyser with an error of approximately $0.6^{\circ}$. Time for analysing one specimen containing 100 grains is approximately three hours at present.

\section{INTRODUCTION}

Ice fabric is essentially important for studies of various phenomena in which polycrystalline ice is involved. Its determination so far has been carried out by an optical method using a Rigsby type universal stage. However, since this method requires laborious work and determines only c-axis orientations with a rather low accuracy, development of a new method has been desired by many investigators.

The X-ray Laue method has an advantage over the optical one in that both c- and a-axes of an ice crystal can be simultaneously determined with high accuracy. However, this method has not been used in ice fabric study until recently, because of the difficulty in determining crystal axes by the analysis of the Laue pattern obtained. The first computer program for automatic analysis of Laue patterns, developed by Ohba, Uehira and Hondoh (1981), was to select a reciprocal lattice vector (RLV) for a spot in order to maximize the probability of coincidence of the measured angle between any pair of diffraction spots with those calculated from the theoretically allowable RLVs. Although this program works very well, it requires several tens seconds of computer time for an analysis of one grain, owing to complex processes in the selection of the RLV.

In the present study we have developed (1) a new program which requires only $0.1 \mathrm{~s}$ of computer time for an analysis of one Laue pattern, (2) a position detector of X-ray Laue spots by which we can precisely determine their coordinates without taking photographs, and (3) an automatic positioning stage for successive irradiation of an X-ray beam on different grains of a polycrystalline thin specimen. Details of the above will be described below.

\section{THE AUTOMATIC ANALYSIS OF X-RAY LAUE PATTERNS OF ICE}

To reduce computer time, the computer program was improved to index zone axes instead of individual spots of a Laue pattern. A zone axis is a common axis involved in many lattice planes of which Laue spots are on a certain conic section appearing in the Laue pattern (see Figure 5b). Since candidates of indices for a zone axis are limited to a small number of low-index directions, this improvement reduces the computer time considerably compared to the former method.
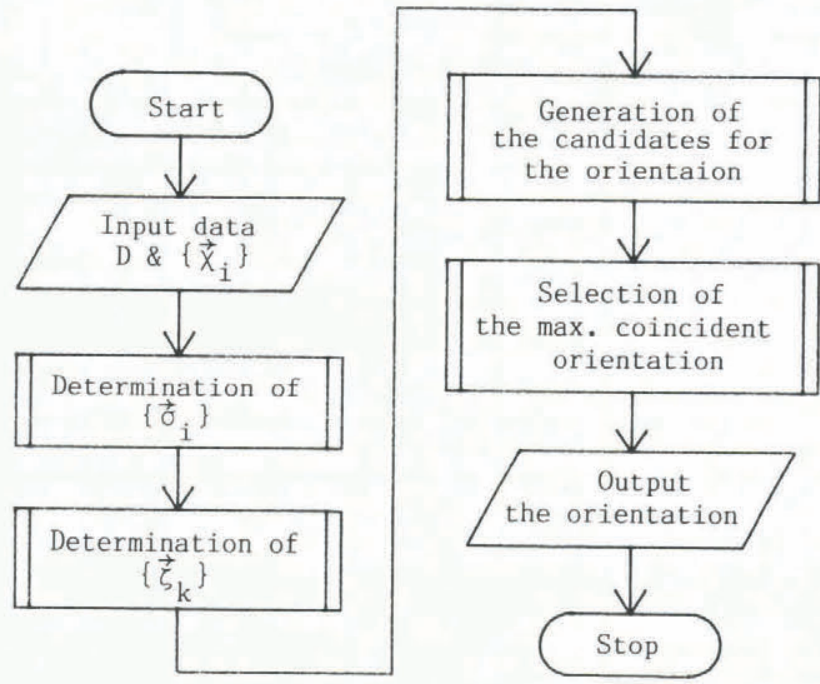

Fig.1. Flow chart of the ALPI.

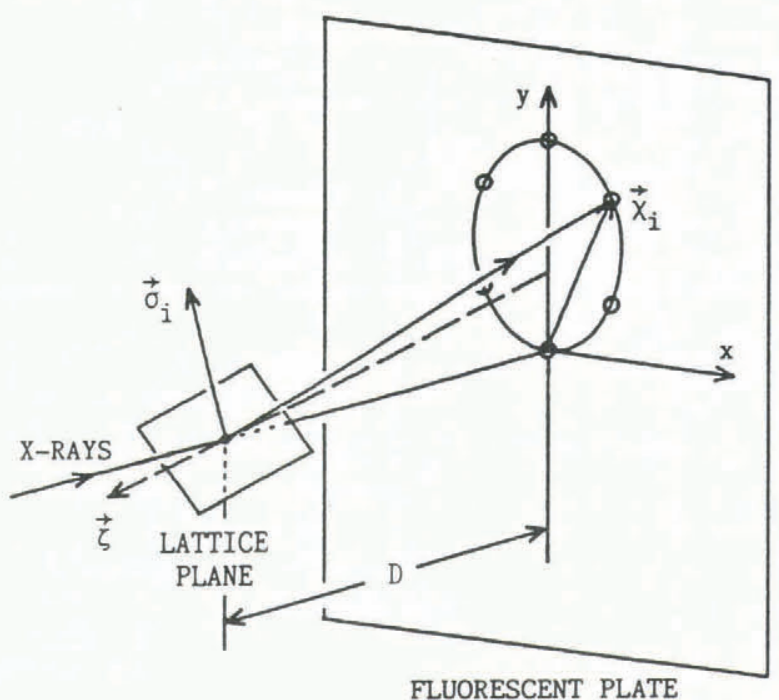

Fig.2. Schematic representation of the formation of Laue spots and the zone axes $\zeta$.

Figure 1 shows a flowchart of the revised program and the main procedures can be summarised as follows:

(1) From a given coordinate on an $i$ th Laue spot, $\vec{x}_{i}=$ $\left(x_{i}, y_{i}\right)$, and a distance (D) between an ice crystal and a film (or a fluorescent plate), the unit vector $\left(\vec{\delta}_{i}\right)$ normal to the lattice plane diffracting $\mathrm{X}$-rays to the spot is determined with respect to the coordinate fixed on the fluorescent plate (see Figure 2). 
(2) Vector products $\left(\vec{y}_{i j}\right)$ are calculated as $\vec{y}_{i j}=\vec{b}_{i} \times \vec{b}_{j}$ for any pair of the plane normals. Then, the zone axis to which both $\vec{\delta}_{i}$ and $\vec{\delta}_{j}$ belong is parallel to $\vec{y}_{i j}$. Since many lattice planes belong to one zone axis, several vectors $\vec{y}_{\mathrm{ij}}$ among those calculated for all pairs of $\vec{b}_{i}$ and $\vec{\sigma}_{j}$ can be identified in one group in which all of $\vec{y}_{j j}$ are mutually parallel within a measurement error of the angle. In practice, there appear only few groups of different $\vec{y}_{i j}$ corresponding to different zone axes. In the present program, the zone axis $\zeta$ to be indexed is determined by averaging the vectors $\vec{y}_{i j}$ in each group.

(3) The determined zone axes $\zeta$ are indexed by comparing the angle between two $\vec{\zeta}$ with the angles calculated from theoretically expected zone axes: [0001], $\langle 1120\rangle,\langle 1123\rangle$ and $\langle 5 \overline{4} 10\rangle$ in the present program.

(4) Using the indexed zone axes, the orientation of the ice crystal is expressed with respect to the coordinate fixed on the fluorescent plate.

The present program, named ALPI (the Automatic analysis of X-ray Laue Patterns of Ice), performs all above procedures within $0.1 \mathrm{~s}$ when it runs on the $\mathrm{M}-280 \mathrm{H}$ (HITACHI) computer. To evaluate the correctness of the above method, we analysed by the use of ALPI many artificial Laue patterns simulated by a computer. Simulations were performed by adding some random errors to the true coordinates of Laue spots for various crystal orientations. The probability of correct determination of the orientation by ALPI critically depended on the errors added to the spot coordinates, and reached $90 \%$ when the errors were smaller than 1 $\mathrm{mm}$ for $100 \mathrm{~mm}$ of $\mathrm{D}$.

THE AUTOMATIC ICE FABRIC ANALYSER (AIFA)

The whole system of the AIFA developed is shown schematically in Figure 3 and as a photograph in Figure 4. This system is composed of two parts, the position detector of X-ray Laue spots of which details are given below, and the automatic positioning stage equipped with crossed polaroids and TV camera.

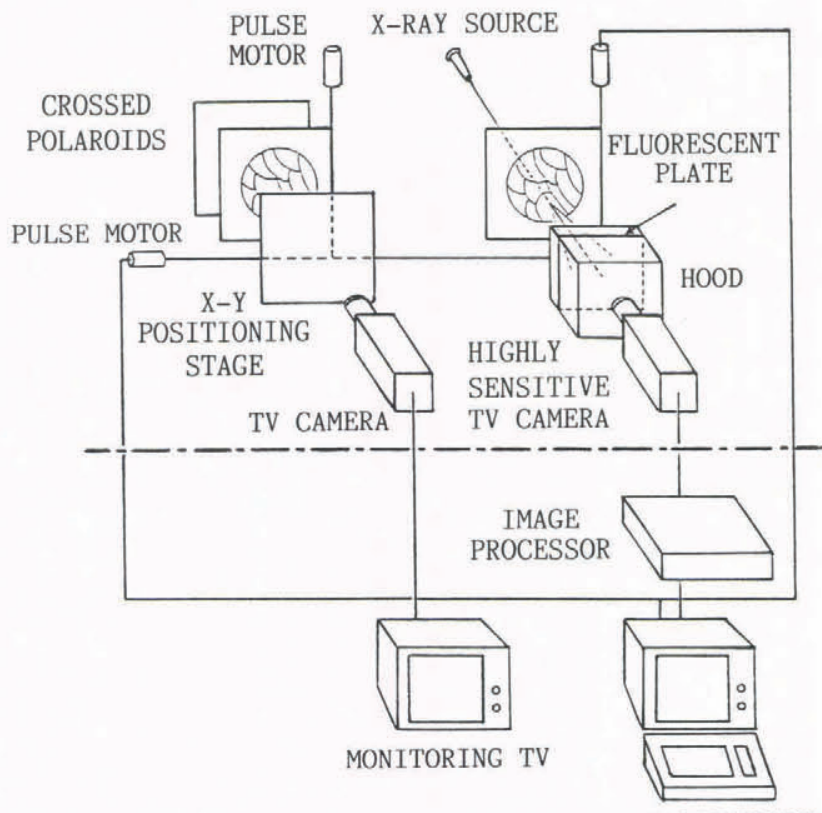

MICROCOMPUTER

Fig.3. Schematic diagram of the AIFA.

THE POSITION DETECTOR OF X-RAY LAUE SPOTS

To measure the coordinates of Laue spots precisely and quickly, we adopted a fluorescent plate with highly sensitive TV camera (SIT tube) instead of X-ray films currently used in the ordinary Laue method. The detector equipment is shown in the right half of Figure 3 and in Figure 4. Even when a powerful $\mathrm{X}$-ray source with a rotating Mo-target was used (RIGAKU RU-200, $60 \mathrm{kV}$ and $200 \mathrm{~mA}$ ) for a specimen of $1 \mathrm{~mm}$ thickness,

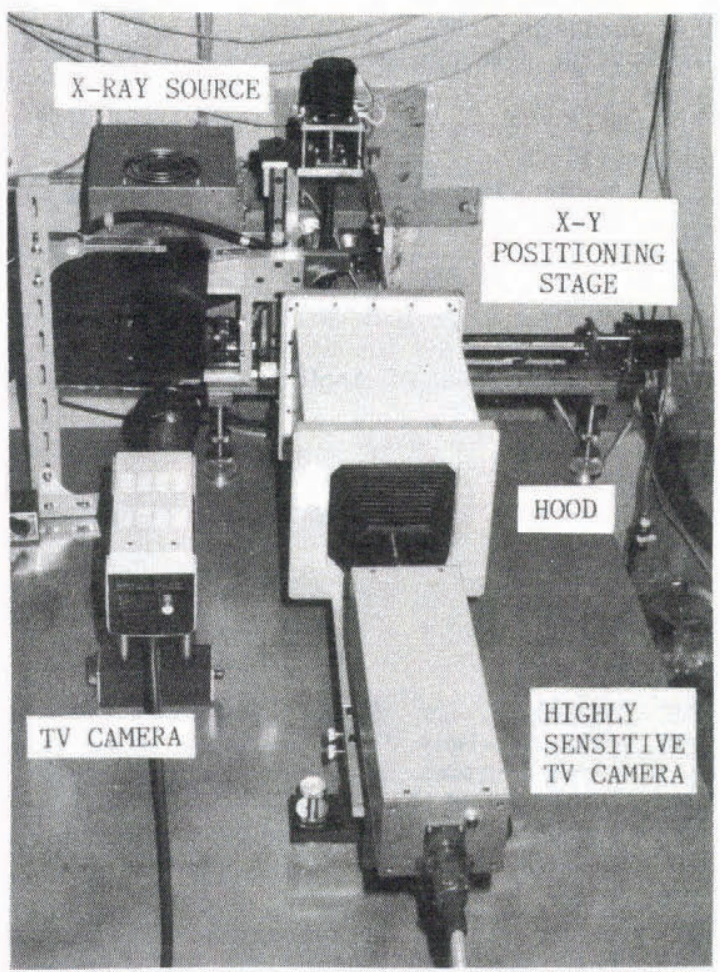

(a)
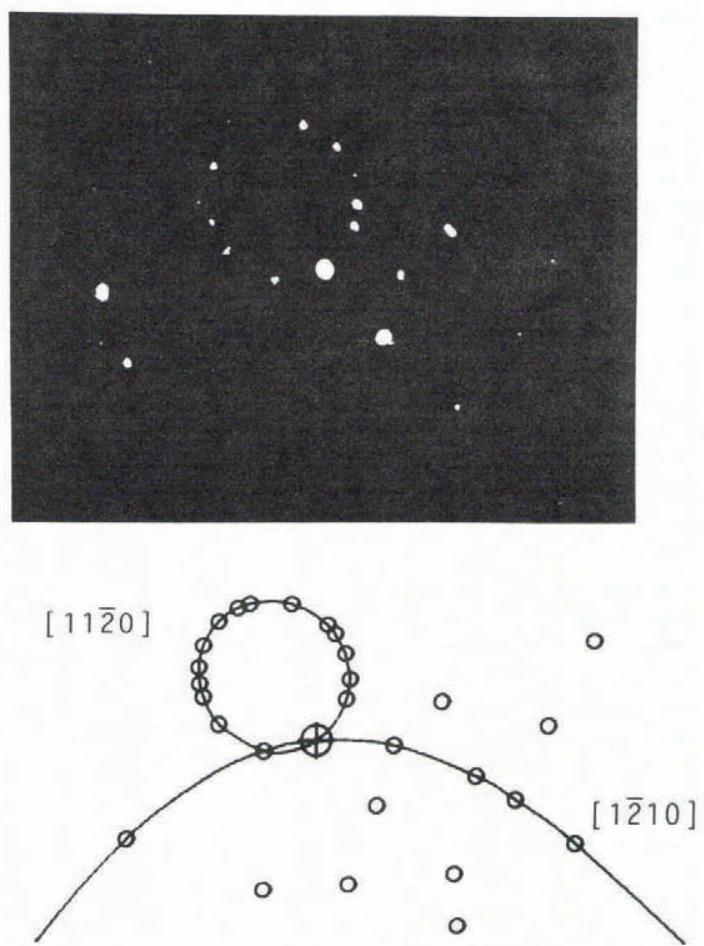

Fig.4. A photograph of the analysis: (a) accumulated image of a Laue pattern; (b) simulated Laue pattern from the crystal orientation determined by the ALPI from the data deduced from (a).

the direct image on the fluorescent plate is very faint. Therefore, TV signals of direct images are accumulated (or integrated) for several seconds in the digital image processor (RIGAKU MJ510FL) to obtain bright image contrasts. An example of Laue spots photographed on the TV screen is shown in Figure 5a. Coordinates of the bright areas on the image processor are transfered to the microcomputer (FUJITSU FM-11) and the coordinate of the center of each bright area was determined to be 
that of a Laue spot. Thus, the $\mathrm{X}$-ray Laue pattern is converted into the coordinate data of the Laue spots which are input to the program ALPI.

Figure $5 \mathrm{~b}$ shows a Laue pattern simulated by using crystal orientation determined by the program ALPI from data of Laue spots in Figure 5a. Two zone axes determined by the program are shown as ellipses in Figure $5 \mathrm{~b}$. Coincidence of Figure $5 \mathrm{a}$ with Figure $5 \mathrm{~b}$ is considered good. From the minute difference of the coordinates of the Laue spots in Figure 5a and b, error of determining the crystal orientation was estimated approximately $0.6^{\circ}$.

\section{THE OPERATION OF THE AIFA}

Practical procedures of making the fabric diagram by the use of the AIFA are as follows (see Figure 3):

Step 1. Thin specimen $(\mathrm{ca} 1 \mathrm{~mm})$ of polycrystalline

ice is fixed to the $\mathrm{X}-\mathrm{Y}$ positioning stage.

Step 2. Numbering and positioning of individual grains to be examined are carried manually at present, distinguighing grains by transmitted polarized light with an aid of a monitoring TV (left half of Figure 3 ). The grain discrimination could be automated in future.

Step 3. The specimen is moved to the $\mathrm{X}$-ray beam, and automatically positioned so that the beam successively irradiates individual grains, positions of which have already been determined in Step 2. After positioning the individual grains, each spot of the Laue pattern is accumulated in the image processor, and the coordinates of the brightness of the image are transfered to the microcomputer.

Step 4. Coordinates $\vec{x}_{i}$ of the Laue spots for individual grains, from brightness data and coordinate data are determined. Determined coordinates $\overrightarrow{\mathrm{X}}_{\mathrm{i}}$ are output to a floppy disk through the microcomputer.

Step 5. $\quad \overrightarrow{\mathrm{x}}_{\mathrm{i}}$ data are transferred to the computing center; crystal axes of individual grains are determined by using program ALPI.

Step 6. Fabric diagrams (both c- and a-axes) by the combination of data obtained in Step 5 and Step 2 and the misorietation relationship between adjacent grains, are obtained.

Total procedures (Step 2 to 5) of analysing one specimen containing 100 grains require approximately three hours: $25 \mathrm{~min}$ for Step 2, $130 \mathrm{~min}$ for Step 3,20 $\min$ for Step4, and $5 \mathrm{~min}$ for Step 5. Although this is at present longer than that required for the Rigsby-type stage operated by a skilled researcher or technician, the time for Step 3 could be reduced considerably if we could use a more powerful computer system for the data acquisition.

\section{REFERENCES}

Cullity B D 1956 Elements of $X$-ray diffraction. Reading, Massachusetts, Addison-Wesley Publishing Co

Ohba R, Uehira I, Hondoh T 1981 Computer-aided spot indexing for $\mathrm{X}$-ray Laue patterns. Japanese Journal of Applied Physics 20(5): 811-816 